\title{
种子植物的选择性败育及其进化生态意义
}

\author{
赵学杰 谭敦炎 \\ (新疆农业大学林学院, 乌鲁木齐 830052)
}

\begin{abstract}
摘 要 种子植物的选择性败育是指植株在花粉源、传粉次序、果实在植株上的位置和发育果实中的种子数目等 因素或者这些因素综合作用的基础上对发育中的幼果或种子选择性败育的现象。植株可以选择性地败育位于果 序顶部或基部的果实以及位于果实基部、中部或柱头端的种子。此现象在被子植物中比较普遍 特别是在豆科、十 字花科和紫草科中最为常见。导致植物选择性败育的主要原因主要有资源限制和遗传因子两个方面。植物通过 选择性败育部分自交或基因型较差的果实或种子，不仅可以提高母本和后代的适合度，而且还可以提高果实或种 子的扩散效率。因此, 对选择性败育的研究在深入了解植物的结实结籽格局、探讨其进化式样与机制等方面具有 重要意义。该文系统总结了国际上有关植物选择性败育的研究工作, 重点介绍了选择性败育发生的式样、导致选 择性败育的因素、选择性败育的进化生态意义, 以及目前研究选择性败育现象的主要方法, 并对该领域今后研究前 景进行了展望。
\end{abstract}

关键词 选择性败育 适合度 结实结籽格局 进化生态学

\section{SELECTIVE ABORTION AND ITS EVOLUTIONARY, ECOLOGICAL SIGNIFI- CANCE IN SEED PLANTS}

\author{
ZHAO Xue-Jie and TAN Dun-Yan * \\ College of Forestry Sciences , Xinjïang Agricultural University , Urumqi 830052 , China
}

\begin{abstract}
Selective abortion in seed plants means selective abortion of developing fruits/seeds based on pollen source, order of pollination, location of fruits on plant, number of developing seeds, or some combination of these. In species with linearly arranged ovules, such as occurs in members of Fabaceae, embryos at the peduncular, stigmatic, or both ends of the fruit can be selectively aborted. Position of fruit within the inflorescence also has a marked effect on abortion. Selective abortion has been documented in many species of Fabaceae , Brassicaceae and Boraginaceae. The primary factor causing selective abortion of fruit and/or seeds is resource limitation. Competition for limited maternal resources occurs among fruits and seeds. Thus, those that are initiated first and have the faster rate of growth can capture more maternal resources and thus are less likely to be aborted than those that are initiated later.

Genetically correlated factors also can result in selective abortion. In some species , fruits from cross-pollinated flowers tend to have more seeds that are more likely to mature than fruits from self-pollinated flowers. Methods used to investigate selective abortion include 1) removing a fraction of the ovules at an early stage ,2) hand-thinning some young fruits or flowers, and 3) using molecule techniques of RAPD and isoenzyme analyses. Selective abortion can increase offspring quality. Progenies that are selectively matured have higher vigor , germinate to higher percentages , produce more leaves , inflorescences and flowers, and mature more seeds than those that are randomly matured. Aborting some seeds also can reduce wing load ( ratio of fruit weight to fruit surface area), improve dispersal distance, and decrease sibling rivalry. Thus, selectively aborting some selfed and/or inferior genotype fruits/seeds increases the fitness of the maternal plant, its offspring, or both. Therefore, research on selective abortion is important to understanding patterns of fruit-set and/or seed-set and its evolutionary pattern and mechanism in angiosperms.
\end{abstract}

We review recent advances in selective abortion research in angiosperms, with emphasis on 1) patterns of selective abortion ;2) factors affecting selective abortion ;3) evolutionary, ecological significance of selective abortion ; and 4) primary methods used to investigate selective abortion. We also discuss prospects for further 
research.

Key words selective abortion ; fitness ; pattern of fruit-set and/or seed-set ; evolutionary ecology

种子植物有性生殖过程中的选择性败育 (Selective abortion) 是指植株在花粉源 (Pollen source)、传粉 次序、果实在植株上的位置和发育果实中的种子数 目等因素或者这些因素综合作用的基础上对发育中 的果实或种子进行选择性败育的现象 (Stephenson， 1981 ; Lee \& Bazzaz, 1982 ; Sutherland \& Delph, $1984 ）$,在种子植物中十分普遍。该现象是由达尔文 在 1876 年首先发现并提出来的, 后来引起众多植物 学家和进化生物学家的广泛关注 (Darwin, 1876 ; Stephenson , 1981 ; Bawa \& Webb , 1984 ; Sutherland , 1986 ; Casper , 1988 ; Lee , 1988 ; Marshall \& Ellstrand , 1988 ; Kozlowski \& Stearns , 1989 ; Vaughton , 1993 ; Melser \& Klinkhamer ,2001; Korbecka et al .,2002 ; 肖 宜安等, 2006)。

在种子植物的结实结籽格局中, 果实与种子的 败育可分为随机败育 (Random abortion) 和选择性败 育 (Nonrandom abortion) 两大类。前者是指植株在发 育的果序或果实中败育一部分果实或种子, 并且这 种败育与它们在果序或果实中的位置以及果实中种 子的数目无关, 可能是由资源限制(Willson \& Price , 1980 ; Lee \& Bazzaz , 1982 ; de Jong \& Klinkhamer, 1989 ; Vaughton , 1993 ; Arathi et al . , 1999)、同胞竞 争( Ganeshaiah \& Uma Shaanker, 1988 ; Arathi et al. , 1996)和花粉限制 (Moody-Weis \& Heywood , 2001 ; Eynard \& Galetto, 2002) 等因素引起的; 后者则是指植 株上果实或种子败育的位置是有一定规律的 (Lee \& Bazzaz, 1982 ; Bawa \& Webb , 1984 ; Casper, 1984 ; Sutherland \& Delph , 1984 ; Casper, 1988 ; Vaughton , $1993 ）$ 植物通过选择性败育可去除不良基因型的后 代 将释放出的资源分配给结合了父母双方优良基 因的后代，从而可提高母本及后代的适合度，因而被 看作是植物的一种进化机制( Casper et al. , 1992)。

目前, 植物选择性败育是国际上植物结实结籽 格局研究中的一个热点问题 (Bawa \& Webb，1984; Sutherland，1986；Lee，1988），并已成为植物进化生 物学研究的一个重要方面( Casper, 1984 ; Sutherland, 1986 ; Melser \& Klinkhamer，2001；Burd，2004）,但我 国在该领域的研究报道还很少(王迎春等，2001; 肖 宜安等, 2006)。为此, 我们在查阅大量相关文献的 基础上，对国内外有关种子植物选择性败育的研究 进展进行了综合分析, 以期为进一步研究植物的结
实结籽格局、探讨其进化式样与机制提供重要的理 论依据。

\section{1 选择性败育的类型}

\section{1 果实的选择性败育}

果实的选择性败育有两种类型。第一类为果序 顶部的果实败育, 即离主轴最近的花比位于花序中 部或远端的花成熟更多的果实。如在紫草科的 Cryptantha flava (Casper , 1984)、蓝䔡 (Echium vulgare) (Nicholls , 1987)、兰科的 Myrosmodes cochleare (Berry \& Calvo，1991)、山龙眼科的 Banksia spinulosa (Vaughton，1993)和十字花科的葱芥 (Alliaria petiola$t a$ ) (Susko \& Lovett-Doust，1998)等植物中，果序从基 部到顶部结实率逐渐下降, 果实内种子数目也逐渐 减少, 且子房和成熟果实的长度也有规律地缩短。 第二类为果序基部的果实败育。如茑尾科的 Iris fulva 存在类似顶端效应的结实格局, 花序顶部的果 实产生激素抑制自顶部向下的果实发育，从而导致 顶端的花具有最高的结实率, 而基部的花结实率最 低(Wesselingh \& Arnold, 2003)。

\section{2 种子的选择性败育}

种子的选择性败育存在 3 种类型。第一类为选 择果实花梗端或基部的种子败育。在此位置的胚珠 受精的可能性较低, 很可能在种子发育的初期败育。 这种式样在 Bauhinia ungulata (Webb \& Bawa , 1985 ; Mena-Alí \& Rocha ,2005a , 2005b)、Chamaecytisus palmensis (Webb \& Shand, 1985 ;Lee \& Bazzaz , 1986)、 Dalbergia sissoo (Ganeshaiah \& Uma Shaanker, 1988 ; Bawa \& Buckley , 1989)、 Cajauns cajan (Cumaraswamy \& Bawa , 1989)、Phaseolus coccineus (Rocha \& Stephenson , 1990)和 Pongamia pinnata (Arathi et al. , 1999) 等植 物中比较常见。第二类为选择性地败育果实柱头端 和基部的种子而成熟果实中部的种子,如豆科的 Pithecellobium saman (Bawa \& Buckley，1989) 和十字 花科的 Brassica oleracea var. botrytis (Gurusamy， 1999）果实中部的胚珠比柱头端和基部的胚珠产生 更多成熟的种子。第三类为选择性地败育果实柱头 端的种子，如在豆科的 Lupinus nanus (Horovitz et al. , 1976)、Lathyrus sylvestris、L. latifolius (Hossaert \& Valéro，1988) 和十字花科的野萝卜 ( Raphanus raphanistrum) ( Marshall \& Ellstrand, 1988 ) 和葱芥 
(Susko \& Lovett-Doust，1998) 等植物中, 果实从基部 到顶端的败育胚珠数目逐渐增加。

另外，在果实内种子非线性排列的植物中，选择 性种子败育发生的位置不是固定的，如在紫草科的 Cryptantha flava 中，同一子房产生的 4 个胚珠排列 在同一个平面上, 这 4 个胚珠的选择性成熟是基于 其活力而不是位置(Casper，1988)。

选择性败育在种子植物中是一种比较普遍的现 象。据对有关文献的统计, 迄今已在豆科、紫草科、 十字花科等 29 个科共 74 种植物中发现存在选择性 败育现象 (表 1 )。

\section{2 选择性败育的原因分析}

\section{1 花的开放顺序}

在植株上不同时间开放的花在资源竞争上存在 着时间差异，植株会对不同时间开放的花进行选择， 通常植株上最先开放的花最可能产生成熟的果实。 Guitian (1994)发现，在蓄薇科植物 Prunus mahaleb 的 花序中最早开放的花结实率可达 $60 \%$ ，最后开放的 花结实率为 0 。在 Pancratium maritimus 中，一个花序 中最早开放的花最可能结实, 且比晚开放的花能产 生更多的种子, 说明最早开放的花可能夺取更多的 母本资源 (Medrano et al. ,2000)。

\section{2 花粉源}

花粉源对植株的结实有显著影响，不同的花粉 源为植物的传粉受精提供了可选择的空间。与由异 花花粉受精产生的果实相比,由自花粉受精产生的 果实中种子数目较少、种子败育率更高、后代活力也 较低(Stephenson，1981)，因此,植物通常会选择异花 粉来完成受精，而选择性败育含有自花粉种子的果 实，以避免近交衰退，提高后代的平均质量和适合 度。如在 Triumfetta semitriloba (Collevatti et al., 1997)、Kalmia latifolia ( Levri , 1998)、Clintonia borealis (Dorken \& Husband , 1999)、 Pinus sylvestris (Kärkkäinen et al ., 1999) 和 Asclepias speciosa (Finer \& Morgan , 2003) 等植物中都存在这种现象。而在缺少花粉供 体选择时, 自花受精的果实也会成熟, 但其后代质量 较低(如 Enterolobium cyclocarpum) (Rocha \& Aguilar, 2001)。Vaccinium myrtillus 同一子房内自交、异交胚 珠都存在时,自交胚珠的相对适合度较低,所结种子 的败育率最高, 且其败育是受基因型制约的, 近交衰 退是败育的主要原因 (Raspé et al., 2004)。在 Wurmbea dioica (Vaughton \& Ramsey , 2003)和 Illicium floridanum (Koehl et al，2004)中, 自花粉果实的败育 也是近交衰退引起的。

Bawa 和 Buckley (1989) 认为,花粉源导致选择 性败育的主要原因是雄配子体间竞争的作用，母本 植株的选择性果实成熟是一个被动过程, 允许在后 代间对有限资源进行竞争，从而去除存活率较低的 后代。在 Asclepias speciosa 中,当父本间竞争激烈 时, 最后成熟的荚果中很大一部分由特定的父本受 精而来(Bookman，1983，1984)。由更优秀的父本产 生的荚果, 其平均种子数、幼苗萌动率及幼苗生物量 均较高。但 Kärkkäinen 等 (1999) 认为, 植物自花粉 受精种子的败育应归因于母本基因型而不是遗传负 荷, 母本的年龄及活力可对种子败育产生一种 母本 环境效应”。“不良母本基因型” ( Bad maternal genotypes)植株比 优秀母本基因型” (Good maternal genotypes) 植株败育更多的果实和种子。此外, Lee (1988)及 Bawa 和 Buckley (1989) 还认为自花粉受精 种子的败育是隐性致死基因表达的结果。

\section{3 花粉量}

沉积于柱头上的花粉供体数目和花粉粒数目也 会影响植株的结实结籽格局。植株不仅在高负荷 (High load)、多供体花粉情况下产生的果实更易成 熟、后代活力更高, 而且高负荷花粉还更容易产生含 有全部种子的果实 (Lee, 1984 ; Quesada et al. , 1996 ; Winsor et al., 2000 )。Cruzan 和 Thomson (1997) 发现, 在 Erythronium grandiflorum 中,与由多 个花粉供体产生的种子相比,由单个花粉供体产生 的种子形成的球茎在一个生长季后平均轻 $5 \%$, 且 3 年后其幼苗具有较低的生活力。在 Lindera benzoin (Niesenbaum , 1996) 和 Mirabilis jalapa (Niesenbaum , 1999)中也发现, 与产生败育果实的花相比, 产生成 熟果实花的花柱中花粉管的数目明显多。

产生上述现象的原因，一方面可能是当柱头上 花粉数目较多时, 植株会选择最高活力、在遗传上优 越的花粉来完成受精, 产生出更高活力的后代 (Niesenbaum，1996，1999），并在果实成熟前选择性 地败育那些基因型较差的果实 (Cruzan \& Thomson, 1997) ;另一方面,高的花粉负荷可刺激母本向这些 果实分配更多的资源, 同时具多数花粉管的花也可 能是受到更多传粉者的拜访，这些花的子房由于较 早受精而发育较早, 从而更具竞争力, 这可能是一种 非遗传的基于花粉管数目的选择性败育 (Niesenbaum , 1996)。 
表 1 存在选择性败育的植物类群

Table 1 Taxa with selective fruit/seed abortion

\begin{tabular}{|c|c|c|c|c|c|}
\hline $\begin{array}{l}\text { 类群 } \\
\text { Taxa }\end{array}$ & $\begin{array}{c}\text { 败育式样 } \\
\text { Patterns of } \\
\text { abortion }\end{array}$ & $\begin{array}{c}\text { 文献 } \\
\text { References }\end{array}$ & 类群 Taxa & $\begin{array}{c}\text { 败育式样 } \\
\text { Patterns of } \\
\text { abortion }\end{array}$ & $\begin{array}{c}\text { 文献 } \\
\text { References }\end{array}$ \\
\hline 龙舌兰科 Agavaceae & & & Myrospermum frutescens & SSA & Bawa \& Webb, 1984; Bawa \& Buckley, 1989 \\
\hline Phormium tenax & SFA & Craig \& Stewart, 1988 & 多花菜豆 Phaseolus coccineus & SSA & Rocha \& Stephenson, 1990 \\
\hline 长瓣丝兰 Yucca baccata & SFA & Addicott \& Bao, 1999 & Pithecellobium saman & SSA & Bawa \& Buckley, 1989 \\
\hline 丝兰 Y. filamentosa & SFA & Marr \& Pellmyr, 2003 & 水黄皮 Pongamia pinnata & SSA & Arathi et al., 1999 \\
\hline Y. kanabensis & SFA & Addicott \& Bao, 1999 & Pterocarpus rohrii & SSA & Bawa \& Webb, 1984; Bawa \& Buckley, 1989 \\
\hline 石蒜科 Amaryllidaceae & & & Pultenaea gunnii & SFA & Burd, 2004 \\
\hline Alstroemeria aurea & SFA & Aizen \& Searcy, 1998 & Senna bicapsularis & SSA & Bawa \& Buckley, 1989 \\
\hline Pancratium maritimum & SFA & Medrano et al., 2000 & S. pallida & SSA & Bawa \& Buckley, 1989 \\
\hline 萝藦科 Apocynaceae & & & Tabebuia rosea & SSA & Bawa \& Webb, 1984 \\
\hline Asclepias speciosa & SFA & Bookman, 1983, 1984; Finer \& Morgan, 2003 & 壳斗科 Fagaceae & & \\
\hline 紫蒇科 Bignoniaceae & & & Quercus ilex & SFA & Díaz et al., 2003 \\
\hline 黄金树 Catalpa speciosa & SFA & Stephenson, 1980 & 金缕梅科 Hamamelidaceae & & \\
\hline 紫草科 Boraginaceae & & & Disanthus cercidifolius & SFA & 肖宜安等, 2006 \\
\hline Cryptantha flava & SFA & Casper, 1984, 1988 & Illiciaceae & & \\
\hline Cynoglossum officinale & SFA & Melser \& Klinkhamer, 2001 & Illicium floridanum & SFA & Vaughton \& Ramsey, 2003 \\
\hline Echium vulgare & SFA & Nicholls, 1987; Klinkhamer et al., 1994 & 鸢尾科 Iridaceae & & \\
\hline 十字花科 Brassicaceae & & & 暗黄㚜尾 Iris fulva & SFA & Wesselingh \& Arnold, 2003 \\
\hline 葱芥 Alliaria petiolate & SFA & Susko \& Lovett-Doust, 1998 & 樟科 Lauraceae & & \\
\hline 甘蓝 Brassica oleracea & SSA & Gurusamy, 1999 & 美国山胡椒 Lindera benzoin & SFA & Niesenbaum, 1996 \\
\hline Raphanus sativus & SSA & Marshall \& Ellstrand, 1988 & 鳄梨 Persea americana & SFA & Degani et al., 1997 \\
\hline Colchicaceae & & & 百合科 Liliaceae & & \\
\hline Wurmbea dioica & SFA & Koehl et al., 2004 & Asphodelus albus & SFA & Obeso, 1993 \\
\hline 山荣英科 Cornaceae & & & 黄花七筋姑 Clintonia borealis & SFA & Dorken \& Husband, 1999 \\
\hline 日本桃叶珊瑚 Aucuba japonica & SFA & Abe, 2001 & 大花猪牙花 Erythronium grandiflorum & SSA & Cruzan \& Thomson, 1997 \\
\hline 葫芦科 Cucurbitaceae & & & 锦葵科 Malvaceae & & \\
\hline Cucurbita foetidissima & SSA & Winsor et al., 2000 & Pavonia dasypetala & SFA & McDade \& Davidar, 1984 \\
\hline 西葫芦 C. pepo & SSA & Quesada et al., 1996 & 芙蓉葵 Hibiscus moscheutos & SFA & Snow \& Spira, 1991 \\
\hline
\end{tabular}


表 1 (续) Table 1 (continued)

\begin{tabular}{|c|c|c|c|c|c|}
\hline C. texana & SSA & Quesada et al., 1996 & 紫茉莉科 Nyctaginaceae & & \\
\hline 杜鹃花科 Ericaceae & & & 紫莱莉 Mirabilis jalapa & SFA & Niesenbaum, 1999 \\
\hline Kalmia latifolia & SFA & Levri, 1998 & 木犀科 Oleaceae & & \\
\hline Vaccinium myrtillus & SSA & Raspé et al., 2004 & Jasminum fruticans & SSA & Thompson \& Dommée, 1993 \\
\hline 豆科 Fabaceae & & & 兰科 Orchidaceae & & \\
\hline Acacia caven & SFA & Torres et al., 2002 & Myrosmodes cochleare & SFA & Berry \& Calvo, 1991 \\
\hline Baptisia leucophaea & SFA & Petersen et al., 1998 & 松科 Pinaceae & & \\
\hline Bauhinia ungulata & SFA、SSA & $\begin{array}{l}\text { Bawa \& Webb, 1984; Webb \& Bawa, 1985; Webb \& } \\
\text { Shand, 1985; Bawa \& Buckley, 1989; Mena-Alí \& } \\
\text { Rocha, 2005b }\end{array}$ & 欧洲赤松 Pinus sylvestris & SSA & Kärkkäinen et al., 1999 \\
\hline Caesalpinia eriostachys & SSA & Bawa \& Webb, 1984; Bawa \& Buckley, 1989 & 山龙眼科 Proteaceae & & \\
\hline 木豆 Cajanus cajan & SSA & Webb \& Shand, 1985; Cumaraswamy \& Bawa, 1989 & Banksia spinulosa & SFA & Vaughton, 1993 \\
\hline Cassia fasciculata & SFA、SSA & Lee \& Bazzaz, 1982, 1986 & Grevillea barklyana & SFA & Harriss \& Whelan, 1993 \\
\hline 长角豆 Ceratonia siliqua & SFA & Arista et al., 1999 & 蓄薇科 Rosaceae & & \\
\hline Chamaecytisus palmensis & SFA、SSA & Webb \& Shand, 1985 & 树唐棣 Amelanchier arbora & SSA & Gorchov, 1988 \\
\hline Cochlospermum vitifolium & SSA & Bawa \& Webb, 1984 & 圆叶樱桃 Prunus mahaleb & SFA & Guitian, 1993, 1994 \\
\hline Dalbergia retusa & SSA & Bawa \& Webb, 1984; Bawa \& Buckley, 1989 & 玄参科 Scrophulariaceae & & \\
\hline 印度黄檀 D. sissoo & SSA & $\begin{array}{l}\text { Ganeshaiah \& Uma Shaanker, 1988; Mohana et al., } \\
2001\end{array}$ & Pedicularis furbishiae & SSA & Menges et al., 1986 \\
\hline Desmodium incanum & SSA & Bawa \& Buckley, 1989 & 椴树科 Tiliaceae & & \\
\hline Enterolobium cyclocarpum & SFA、SSA & Bawa \& Buckley, 1989; Rocha \& Aguilar, 2001 & Triumfetta semitriloba & SFA & Collevatti et al., 1997 \\
\hline Gliricidia sepium & SSA & Bawa \& Buckley, 1989 & 堇菜科 Violaceae & & \\
\hline Lathyrus latifolius & SSA & Hossaert \& Valéro, 1988 & 三色堇 Viola tricolor & SSA & Skogsmyr \& Lankinen, 1999 \\
\hline L. sylvestris & SSA & Hossaert \& Valéro, 1988 & 桃金娘科 Myrtaceae & & \\
\hline Lotus corniculatus & SFA & Stephenson \& Winsor, 1986 & 海南蒲桃 Syzygium cuminii & SSA & Arathi et al., 1996 \\
\hline Lupinus nanus & SSA & Horovitz et al., 1976 & 苼藜科 Zygophyllaceae & & \\
\hline 紫苜宿 Medicago sativa & SSA & Horovitz et al., 1976 & 四合木 Tetraena mongolica & SFA & 王迎春等, 2001 \\
\hline Mimosa pigra & SSA & Bawa \& Buckley, 1989 & & & \\
\hline
\end{tabular}

SFA：选择性果实败育 Selective fruit abortion SSA：选择性种子败育 Selective seed abortion 


\section{4 花粉管生长速率}

花粉管竞争可以影响植株产生的种子数、近交 衰退水平以及由不同异交供体产生的种子适合度。 在竞争条件下, 花粉管生长速率是一个花粉供体产 生种子的良好指示,而且花粉管生长速率随每花柱 中花粉管的数量增加而增加, 这可能归因于生长的 花粉管之间的正相互作用 (Niesenbaum，1999)。由 生长最快的花粉管受精的种子更容易成熟，因为生 长快的花粉管显示了优越的父本基因型,并且最早 受精的胚珠存在对资源竞争的时间优势 (Stephenson ，1981；Lee \& Bazzaz，1986；Snow \& Spira，1991）， 这些胚珠是母本资源的最佳竞争者, 它们更容易被 母本选择性成熟,而且能产生更具活力的后代 ( Bawa \& Webb , 1984 ; Lee, 1984 ; Arathi et al. , 1999 ; Mena-Alí \& Rocha ,2005a)。

异花粉的萌发及花粉管的生长通常比自花粉的 快，如在山龙眼科植物 Grevillea barklyana 中, 异花花 粉管在自花花粉管之前到达胚珠 (Harriss \& Whelan， 1993 ) ,在野萝卜 (Marshall \& Ellstrand , 1988)、Hibiscus moscheutos (Snow \& Spira, 1991 ) 和 Viola tricolor (Skogsmyr \& Lankinen，1999) 等植物中都存在着由花 粉管生长速率不等造成的选择性败育现象。在香踠 豆属的 Lathyrus sylvestris 和 L. latifolius 中, 败育率最 高部位的胚珠由生长最慢、最晚到达子房的花粉管 受精, 这样的胚在竞争母本资源时活力较差 或者最 早形成的胚首先分泌生长激素, 在竞争母本资源时 有时间优势 (Hossaert \& Valéro，1988)。

\section{5 发育中果实内的种子数目}

对于发育中的果实, 含有种子数较多的果实成 熟的可能性高, 而含有种子数目较少的果实容易败 育 (Gorchov，1988；Obeso，1993) 如在 Cassia fasciculata (Lee \& Bazzaz , 1982)、Asclepias speciosa (Bookman , 1983 , 1984)、Cochlospermum vitifolium (Bawa \& Webb , 1984)、 Pavonia dasypetala (McDade \& Davidar , 1984)、 Chamaecytisus palmensis (Webb \& Shand, 1985)、Lotus corniculatus (Stephenson \& Winsor, 1986 )、Triumfetta semitriloba (Collevatti et al . , 1997)和 Ceratonia siliqua (Arista et al ., 1999)中都存在这种现象。

造成这种现象的主要原因, 可能是多种子果实 比少种子果实能更有效地从母本获取资源, 而当资 源有限时，母本会选择含种子数目较少的幼果败育， 以此提高后代的平均质量 (McDade \& Davidar , 1984 ; Collevatti et al. , 1997)。也可能少种子果实中的种 子产生的生长激素太少而不能刺激果实发育成熟
(Petersen et al. , 1998)。

\section{6 虫食作用}

虫食作用对选择性败育发生程度的影响通常 表 现为对营养器官 (叶片) 和繁殖器官 (果实) 的危害。 虫食营养器官 (叶片) 减少了资源积累, 导致繁殖需 求和可利用资源之间不平衡的扩大, 资源的减少会 导致发育果实之间的竞争增强, 并潜在地使败育更 具选择性 (Stephenson , 1981； Menges et al. , 1986 ; Niesenbaum , 1996 ; Holland et al. , 2004 )。如在 Catalpa speciosa 的胚珠受精后去除植株上的部分叶 片来模拟虫食作用，发现在处理枝条上成熟的果实 较小, 果实中的种子既少又小，且果实有显著的败育 (Stephenson, 1980); 在 Lindera benzoin 中,处理枝条 上有 $67.2 \%$ 的果实最后败育 (Niesenbaum, 1996) , 这 些结果表明, 植株会选择性地败育经历虫食的枝条 上的果实，因为去除部分叶片后会导致果实间更激 烈地竞争有限资源, 当少量子房发育时, 植株应选择 性地向高质量的胚分配资源来提高适合度 (Webb \& Bawa , 1985 ; Niesenbaum , 1996)。

为了应对虫食对繁殖器官 (果实) 的危害, 植株 通常会败育部分含有虫卵的果实, 以控制昆虫的种 群数量 (Verdú1 \& García-Fayos，1998；Holland \& DeAngelis，2001），且败育部分果实使可利用资源与 果实产量相匹配, 这样可通过控制果实数目来提高 果实的质量 (Stephenson, 1980)。如在丝兰属植物 Yucca kanabensis 和 Y. baccata 中,传粉昆虫丝兰蛾 (Yucca moth)将卵产在植物的生殖组织中 幼虫以之 为食，二者是互惠共生关系。这两种植物通过选择 性脱落含有虫卵的花来控制丝兰蛾的种群, 但只有 当丝兰蛾产卵直接破坏发育中的胚珠时,才发生选 择性脱落 (Addicott \& Bao，1999)。Marr 和 Pellmyr (2003) 通过模拟丝兰蛾产卵器的插入及产卵部位在 胚珠中位置的变化发现,在丝兰蛾的自然产卵过程 中胚珠受到物理性损伤, 这种物理性损伤导致果实 的选择性脱落。

\section{7 胁迫环境条件}

胁迫环境条件也会影响植物的结实格局。在胁 迫条件下植株缺乏某些资源, 因而会选择性败育生 活力较差的种子或果实来提高适合度。如在干旱胁 迫的野萝卜中, 从果实基部到柱头端的种子重量下 降趋势比对照组更明显, 且柱头端的种子败育率更 高, 说明在胁迫环境下存在位置依赖的资源限制 (Marshall \& Ellstrand, 1988)。在 Cassia fasciculata 中，浇水对果实及种子的败育没有影响，而干旱处理 
可使败育率明显上升, 且其败育是有选择性的 (Lee \& Bazzaz , 1986)。

由以上可以看出，导致植物选择性败育的根本 原因主要有资源限制和遗传因子两个方面。从资源 的角度看, 果实或种子的败育在很大程度上与植株 的源/库比相关 (Lee , 1988 ; Marcelis et al ., 2004)。 当供给繁殖的资源有限时，植株上距光合产物源和 营养源最近的果实或种子存在对资源竞争的空间优 势, 发育较早的果实或种子存在竞争的时间优势, 这 些果实或种子能获取更多的母本资源, 因而更容易 成熟(Wyatt，1980；Wyatt，1982；Casper，1984；Webb \& Bawa, 1985 ; Webb \& Shand, 1985 ; Lee, 1988 ; Vaughton, 1993)。同时, 不同花序以及同一花序的 子房之间也可以竞争或重新分配有限的资源 （Vaughton，1993）植株会选择不同的果实来分配资 源, 如母株会向合子质量高或高花粉负荷产生的果 实分配更多资源（Niesenbaum，1996；Skogsmyr \& Lankinen，2002）。植株通过选择性败育可以保留部 分母本资源或释放出一些资源, 以重新分配给其它 更易成熟的果实或种子, 或用来产生更多的花 (Casper，1984； Nicholls，1987)。一些研究者还认 为 植物不发达的心皮维管组织在胚珠发育过程中 不能及时有效地输送资源也可导致部分胚珠的选择 性败育 (Gorchov，1988; Vaughton，1993)。从遗传的 角度看, 异花受精的含有优秀亲本基因型的果实或 种子更易于成熟(Bookman，1983，1984；Niesenbaum， $1996 ， 1999$; Raspé et al . 2004) ,而含有较差基因型 的果实则在成熟前被选择性地败育 (Cruzan \& Thomson, 1997)。在果实内, 遗传上相关程度较大的胚之 间竞争较小, 败育率较低; 而遗传上相关程度较小的 胚之间竞争激烈, 种子败育率较高 (Uma Shaanker et al. , 1988 ;Mohana et al., 2001)。此外, Charlesworth (1989)认为,胚珠败育应归因于遗传负荷;Bawa 等 (1989)认为, 依据遗传负荷理论, 败育的胚珠不可能 再存活, 但在一些物种中如果占优势地位的胚被破 坏, 那么原本败育的胚就可以发育成熟。

\section{3 选择性败育的进化生态意义}

选择性败育作为种子植物中一种比较常见的现 象 ,其进化生态意义主要体现在以下两个方面：

首先, 选择性败育可以提高母本及后代的适合 度。在自然条件下, 选择性败育通常发生在异交和 自交不亲和的种类中 ( Casper , 1984 ; Sutherland， 1986)。在自交亲和的物种中, 近交提高了纯合性,
降低了种子的遗传变异，从而降低了对果实或种子 选择性败育的基础, 选择性败育的优势会降低并可 能最终消失, 同时自交产生的胚和胚乳质量也要低 于异交的;而在异交或自交不亲和的花中，选择性败 育可去除不良基因型的后代，保存结合了父母双方 优良基因的后代，有利于物种的进化 (Thompson \& Dommée , 1993)。

在自然条件下，植株会首先选择败育品质低劣 的果实或种子，这种选择通常基于种子的基因组成 (Genetic constitution) (Lee \& Bazzaz , 1982 ; Sutherland , 1986 ; Nicholls , 1987 ; Thompson \& Dommée , 1993 ; Niesenbaum , 1996 , 1999 ; Korbecka et al . 2002)。很 多植物首先选择败育自交的胚珠来提高种子的数量 和质量 (Vaughton，1993；Kärkkäinen et al. , 1999 ; Finer \& Morgan , 2003) ，且选择性败育后成熟果实中 的种子比人工疏花或破坏部分胚珠后成熟果实中的 种子萌发率更高 幼苗有更高的活力和繁殖输出 相 对适合度也较高(Bawa \& Webb，1984; Stephenson \& Winsor, 1986 ; Casper, 1988 ; Kozlowski \& Stearns , 1989 ; Andersson , 1990 ; Melser \& Klinkhamer , 2001 ; Burd , 2004 ; Mena-Alí \& Rocha ,2005b)。Burd (1998) 建立了一个有关选择性败育的模型, 认为通过选择 性败育最低质量的后代增加了后代的平均质量, 稍 微过量的花是提高植株适合度的一种策略。通过选 择性败育来提高后代的适合度, 可能是某些生活在 资源贫乏、干旱少雨条件下的植物在长期进化过程 中形成的一种生殖保障(王迎春等 2001)。

其次，选择性败育对果实的扩散具有重要意义。 植物果实内种子的数目可以看作是扩散收益和包装 消耗之间的一种权衡 (Trade-off) (Ganeshaiah \& Uma Shaanker，1988），开花植物果实中种子数目的减少 可被认为是一种进化的倾向, 进化出单种子 (Singleseed)扩散单位在某种程度上是扩散过程中和扩散 后同胞竞争等选择性压力作用的结果 (Casper et $a l$ ，1992)。在果实内败育部分种子可以降低扩散 单位的重量, 扩大传播距离, 同时减少后代间的竞争 (Casper , 1984 ; Uma Shaanker et al. , 1988 )。如 Pongamia pinnata (Arathi et al , 1999) 和 Dalbergia sissoo (Ganeshaiah \& Uma Shaanker, 1988) 的大多数果 实中仅含一粒种子, 通过选择性败育部分种子来降 低翅负荷（Wing load），以提高果实的扩散效率。 Syzygium cuminii 败育一部分种子的果实是对鸟类扩 散的一种适应(Arathi et al. , 1996)。植物果实扩散 式样及其从母本脱离后种子的命运可能是每朵花产 
生种子数目的主要选择因子 (Casper, 1984)。因此, 从扩散意义上讲, 成熟、含有少量种子的果实具有更 高的适合度(Lee，1988)。

\section{4 检测和分析选择性败育的主要方法}

\section{1 破坏部分胚珠}

在具选择性败育现象的植物中, 正常情况下植 株会选择性败育质量较低的胚或种子。在发育初期 人工破坏部分胚珠后, 由于缺少供选择的胚珠 剩余 胚珠的败育率会下降, 原来败育的胚珠会发育为成 熟种子 将这些种子和自然条件下选择性成熟的种 子的活力进行比较, 可以证明选择性败育现象的存 在(Skogsmyr \& Lankinen , 2002)。

Casper (1988)、Melser 和 Klinkhamer (2001) 及 Mena-Alí 和 Rocha (2005b) 分别在 Cryptantha flava、 Cynoglossum officinale 和 Bauhinia ungulate 中发现, 破 坏部分胚珠后剩余胚珠的败育率下降, 通常败育的 胚珠会发育为成熟的种子，原因是破坏部分胚珠后， 植株可利用的资源相对增多, 将剩余的资源分配给 通常情况下败育的胚珠使之发育成熟。但将种子播 种后发现，正常情况下成熟的种子萌发率明显高于 由剩余胚珠发育成的种子, 且幼苗存活率高。以上 结果说明，正常情况下植株选择性成熟具有更高活 力的胚, 败育低质量、活力较低的胚(Casper, 1988; Melser \& Klinkhamer, 2001)。而且 Casper (1988) 认 为, 研究中观测到的种子质量的区别可能是由子房 的不同生理响应 (Physiological responses) 造成的, 而 不是由胚的基因型造成。

\section{2 人工疏花疏果}

人工疏花疏果是许多研究者经常采用的方法。 此法操作方便，容易控制。植株通常会产生过多的 花或者发育过多的果实，人工去除部分花或果实后 观察植株的结实情况, 通过与对照组比较可验证植 株的选择性败育情况。如在 Prunus mahaleb (Guitian , 1993)、P. longiflora (Yang et al. ,2005) 和长柄 双花木(Disanthus cercidifolius) (肖宜安等，2006)中， 人工去除部分花会提高剩余花的结实率，但去花后 植株产生的果实和种子的重量都明显比对照组低。 在 Banksia spinulosa 中去除基部花明显会增加远轴 端花的结实率 (Vaughton , 1993)。在 Pancratium maritimum 中去除早开放的花后, 剩余花的结实率和每 花的平均结籽率都会上升 (Medrano et al ，，2000)。 人工疏果后也存在类似的现象, 将 Catalpa speciosa (Stephenson , 1980) 和 Cassia fasciculata (Lee \& Bazzaz ,
$1982 ， 1986)$ 植株的果序去除部分果实后，几乎所有 剩余的果实都会发育成熟, 且每果实比对照植株产 生更多的胚珠。

以上结果说明: 通过人工疏花疏果限制了植株 对败育果实的选择, 同时降低了果实间对资源的竞 争(Lee \& Bazzaz , 1982，1986) , 使母本将原来要给予 成熟果实的资源分配给剩余果实 (Stephenson, 1980) 致使自然情况下败育的果实发育成熟 (Guitian , 1993)。

\section{3 分子生物学方法}

从已有文献来看, 目前运用分子生物学手段研 究植物果实或种子选择性败育的报道还很少, 已有 的研究主要采用 RAPD 分析、同工酶分析等技术手 段分析种子的 DNA 相关性或者异交程度, 从生化及 分子水平来探究选择性种子败育的原因。如 Mohana 等(2001)利用 RAPD 分析和同工酶技术发现， 在 Dalbergia sissoo 的果实内, 遗传上相关程度较小 的胚之间竞争激烈,造成较高的种子败育率。Degani 等(1997)和 Wang(2003) 也用同工酶技术证明了 Persea americana 和 Fagus sylvatica 选择性败育自交 产生种子的机制。

\section{5 展 望}

选择性败育是一种普遍存在于种子植物中的现 象, 随着研究的不断拓展和深入, 会有更多的类群加 入到此行列中 (Bookman，1984），同时还可能会发现 其它完全不同的选择性败育机制。植物选择性败育 不仅可用来解释许多植物为何会产生过多的花或胚 珠, 而且还是植物选择最高质量后代的一种进化机 制。如前所述, 目前对选择性败育的研究主要集中 在败育发生的位置、诱导因素、对果实扩散的影响以 及在植物进化上的意义等方面 (Casper , 1984 ; Webb \& Bawa , 1985 ; Webb \& Shand, 1985 ; Sutherland, 1986 ; Burd，2004）, 而对于植物选择性败育所涉及 到的繁殖生物学、发育生物学和进化生物学等领域 的具体机制和式样问题, 还有待于在层次上和研究 方法上深入探究。

在研究层次上,目前已有的对选择性败育现象 的研究大多处于定性的观察分析阶段, 对产生该现 象的原因分析大多是推测性的，且不同学者持不同 见解 (Lee \& Bazzaz, 1986 ; Mikesell , 1988 ; Korbecka et $a l ., 2002)$ 。而对植株为何要选择一部分果实或 种子败育而让其它部分成熟、为何有的物种只败育 已传粉的花或仅败育幼果或二者均败育, 以及在对 
不同果实间选择性分配资源时是如何确定后代活力 的等核心问题, 至今缺乏令人信服的解释, 有必要从 群体遗传学、发育生物学和进化生物学角度深入探 讨植物选择性败育的内在调控机制及其进化意义 (Stephenson , 1981 ; Marshall \& Ellstrand , 1988 ; Gurusamy , 1999 ; Díaz et al . , 2003 ; Mena-Alí \& Rocha , 2005b)。

在研究方法上, 目前已有的研究大多借助于常 规的形态学观察手段,有些实验结果可能是人工操 作造成的(如当人工破坏部分胚珠时可能会对其它 胚珠造成损伤而导致其败育) (Casper，1988）,对生 理、生化及分子生物学等研究手段的应用还极少, 今 后应该加强对选择性果实或种子败育现象观察的控 制性试验 (如控制花芽和花的数目、已传粉花的数 目、特定果实的败育、果实的亲本、虫食量等) (Stephenson，1981) , 并运用多种研究手段来进行综 合分析 ( Quesada et al. , 1996 ; Korbecka et al. , 2002), 以减小实验误差, 最大可能地探究植物选择 性败育的本质。

\section{参 考 文 献}

Abe T (2001) . Flowering phenology, display size, and fruit set in an understory dioecious shrub, Aucuba japonica (Cornaceae). American Journal of Botany, 88, 455 - 461.

Addicott JF, Bao T (1999) . Limiting the costs of mutualism: multiple modes of interaction between yuccas and yucca moths. Proceedings of the Royal Society of London Series B: Biological Sciences, 266, $197-202$.

Aizen MA, Searcy KB (1998). Selective fruit filling in relation to pollen load size in Alstroemeria aurea (Alstroemeriaceae). Sexual Plant Reproduction, 11, $166-170$.

Andersson S (1990). No evidence for selective seed maturation in Anchusa officinalis (Boraginaceae). Oikos, 57, 88-93.

Arathi HS, Ganeshaiah KN, Uma Shaanker R, Hegde SG (1996) . Factors affecting embryo in Syzygium cuminii (L.) Skeels (Myrtaceae). International Journal of Plant Sciences, 157, 49-52.

Arathi HS, Ganeshaiah KN, Uma Shaanker R, Hegde SG (1999) . Seed abortion in Pongamia pinnata (Fabaceae). American Journal of Botany, 86, 659-662.

Arista M, Ortiz PL, Talavera S (1999). Apical pattern of fruit production in the racemes of Ceratonia siliqua (Leguminosae: Caesalpinioideae) : role of pollinators. American Journal of Botany, $86,1708-1716$.

Bawa KS, Ganeshaiah KN, Uma Shaanker R (1989). Embryo and seed abortion in plants. Nature, 342, 625-626.

Bawa KS, Webb CJ (1984). Flower, fruit and seed abortion in tropical forest trees: implications for the evolution of paternal and maternal reproductive patterns. American Journal of Botany, 71,
$736-751$.

Bawa KS, Buckley DP (1989). Seed: ovule ratios, selective seed abortion, and mating systems in Leguminosae. Monographs in Systematic Botany from the Missouri Botanical Garden, 29, 243 262 .

Berry PE, Calvo RN (1991). Pollinator limitation and position dependent fruit set in the high Andean orchid Myrosmodes cochleare (Orchidaceae). Plant Systematics and Evolution, 174, 93 101 .

Bookman SS (1983). Costs and benefits of flower abscission and fruit abortion in Asclepias speciosa. Ecology, 64, 264-273.

Bookman SS (1984). Evidence for selective fruit production in Asclepias. Evolution, 38, $72-86$.

Burd M (1998)." Excess" flower production and selective fruit abortion: a model of potential benefits. Ecology, 79, 2123 2132 .

Burd M (2004). Offspring quality in relation to excess flowers in Pultenaea gunnii (Fabaceae). Evolution, 58, 2371 - 2376.

Casper BB (1984). On the evolution of embryo abortion in the herbaceous perennial Cryptantha flava. Evolution, 38, 1337 1349 .

Casper BB ( 1988$)$. Evidence for selective embryo abortion in Cryptantha flava. American Naturalist, 132, 318 - 326.

Casper BB, Head SB, Apanius V (1992). Ecological correlates of single-seededness in a woody tropical flora. Oecologia, 90, $212-217$.

Charlesworth D (1989). Evolution of low female fertility in plants: pollen limitation, resource allocation and genetic load. Trends in Ecology \& Evolution, 4, 289 - 292.

Collevatti RG, Amaral MEC, Lopes FS (1997). Role of pollinators in seed set and a test of pollen limitation hypothesis in the tropical weed Triumfetta semitriloba (Tiliaceae). Revista de Biología Tropical, 45, $1401-1407$.

Craig JL, Stewart AM (1998) . Reproductive biology of Phormium tenax: a honeyeater-pollinated species. New Zealand Journal of Botany, 26, $453-463$.

Cruzan MB, Thomson JD (1997). Effects of pre-dispersal selection on offspring growth and survival in Erythronium grandiflorum. Journal of Evolutionary Biology, 10, 295 - 314.

Cumaraswany A, Bawa KS (1989). Sex allocation and mating systems in pigeonpea, Cajanus cajan (Fabaceae). Plant Systematics and Evolution, 168, 59-69.

Darwin CR (1876). The Effects of Cross- and Self-fertilization in the Vegetable Kingdom. John Murray, London, 398-400.

Degani C, El-Batsri R, Gazit S (1997). Outcrossing rate, yield, and selective fruit abscission in "Ettinger" and" Ardith" avocado plots. Journal of the American Society for Horticultural Science, $122,813-817$.

Díaz M, Møller AP, Pulido FJ (2003). Fruit abortion, developmental selection and developmental stability in Quercus ilex. Oecologia, $135,378-385$. 
Dorken ME, Husband BC (1999). Self-sterility in the understory herb Clintonia borealis (Liliaceae). International Journal of Plant Sciences, 160, 557 - 584 .

Eynard C, Galetto L (2002). Pollination ecology of Geoffroea decorticans (Fabaceae) in central Argentine dry forest. Journal of Arid Environments, 51, $79-88$.

Finer MS, Morgan MT (2003). Effects of natural rates of geitonogamy on fruit set in Asclepias speciosa (Apocynaceae) : evidence favoring the plant's dilemma. American Journal of Botany, 90, 1746 - 1750 .

Ganeshaiah KN, Uma Shaanker R (1988). Seed abortion in winddispersed pods of Dalbergia sissoo : maternal regulation or sibling rivalry? Oecologia, 77, $135-139$.

Gorchov DL (1988). Effects of pollen and resource on seed number and other fitness components in Amelanchier arborea (Rosaceae: Maloideae) . American Journal of Botany, 75, 1275 - 1285.

Guitian J (1993). Why Prunus mahaleb (Rosaceae) produces more flowers than fruits. American Journal of Botany, 80, 1305 1309 .

Guitian J ( 1994 ). Selective fruit abortion in Prunus mahaleb (Rosaceae) . American Journal of Botany, 81, 1555-1558.

Gurusamy C (1999). The pattern of seed formation in cauliflower (Brassica oleracea L. var. botrytis) with special reference to seed abortion. Canadian Journal of Botany, 77, 1189-1192.

Harriss F, Whelan RJ (1993). Selective fruit abortion in Grevillea barklyana (Proteaceae). Australian Journal of Botany, 41, $499-509$.

Holland JN, DeAngelis DL (2001) . Population dynamics and the ecological stability of obligate pollination mutualisms. Oecologia, $126,575-586$.

Holland JN, Bronstein JL, DeAngelis DL (2004) . Testing hypotheses for excess flower production and low fruit-to-flower ratios in a pollinating seed-consuming mutualism. Oikos, 105, 633-640.

Horovitz A, Meiri L, Beiles A (1976). Effects of ovule positions in fabaceous flowers on seed set and outcrossing rates. Botanical Gazette, 137, 250 - 254 .

Hossaert M, Valéro M (1988). Effect of ovule position in the pod on patterns of seed formation in two species of Lathyrus (Leguminosae: Papilionoideae). American Journal of Botany, 75, 1714 - 1731 .

de Jong TJ, Klinkhamer PGL (1989) . Limiting factors for seed production in Cynoglossum officinale. Oecologia, 80, $167-172$.

Kärkkäinen K, Savolainen O, Koski V (1999). Why do plants abort so many developing seed: bad offspring or bad maternal genotypes? Evolutionary Ecology, 13, 305 - 317.

Klinkhamer PGL, de Jong TJ, Nell HW (1994) . Limiting factors for seed production and phenotypic gender in the gynodioecious species Echium vulgare (Boraginaceae). Oikos, 71, $469-478$.

Koehl V, Thien LB, Heij EG, Sage TL (2004). The causes of self-sterility in natural populations of the relictual angiosperm, $I l$ licium floridanum (Illiciaceae). Annals of Botany, 94, 43-50.
Korbecka G, Klinkhamer PGL, Vrieling K (2002). Selective embryo abortion hypothesis revisited-a molecular approach. Plant Biology, 4, 298 - 310 .

Kozlowski J, Stearns SC (1989). Hypotheses for the production of excess zygotes: models of bet-hedging and selective abortion. Evolution, 43, 1369 - 1377.

Lee TD (1984) . Patterns of fruit maturation: a gametophyte competition hypothesis. American Naturalist, 123, 427 - 432 .

Lee TD (1988). Patterns of fruit and seed production. In: LovettDoust J, Lovett-Doust L eds. Plant Reproductive Ecology. Oxford University Press, New York, 179 - 202.

Lee TD, Bazzaz FA (1982). Regulation of fruit and seed production in an annual legume, Cassia fasciculata. Ecology, 63, 1363 1373 .

Lee TD, Bazzaz FA (1986). Maternal regulation of fecundity: nonrandom ovule abortion in Cassia fasciculata Michx. Oecologia, $68,459-465$.

Levri MA (1998). The effect of timing of pollination on the mating system and fitness of Kalmia latifolia (Ericaceae). American Journal of Botany, 85, 1626 - 1630 .

Marcelis LFM, Heuvelink E, Baan Hofman-Eijer LR, den Bakker J, Xue LB (2004). Flower and fruit abortion in sweet pepper in relation to source and sink strength. Journal of Experimental Botany, 55, $2261-2268$.

Marr DL, Pellmyr O (2003). Effect of pollinator-inflicted ovule damage on floral abscission in the yucca-yucca mothmutualism: the role of mechanical and chemical factors. Oecologia, 136, $236-243$.

Marshall DL, Ellstrand NC (1988). Effective mate choice in wild radish: evidence for selective seed abortion and its mechanism. American Naturalist, 131, 739 - 756.

McDade LA, Davidar P (1984). Determinants of fruit and seed set in Pavonia dasypetala (Malvaceae). Oecologia, 64, 61-67.

Medrano M, Guitión P, Guitión J (2000). Patterns of fruit and seed set within inflorescences of Pancratium maritimum (Amaryllidaceae) : nonuniform pollination, resource limitation, or architectural effects? American Journal of Botany, 87, 493 - 501 .

Melser C, Klinkhamer PGL (2001). Selective seed abortion increases offspring survival in Cynoglossum officinale (Boraginaceae). American Journal of Botany, 88, 1033 - 1040 .

Mena-Alí JI, Rocha OJ (2005a) . Effect of ovule position within the pod on the probability of seed production in Bauhinia ungulate (Fabaceae). Annals of Botany, 95, 449-455.

Mena-Alí JI, Rocha OJ (2005b) . Selective seed abortion affects the performance of the offspring in Bauhinia ungulata. Annals of Botany, 95, 1017 - 1023 .

Menges ES, Waller DM, Gawler SC (1986). Seed set and seed predation in Pedicularis furbishiae, a rare endemic of the ST. John River, Maine. American Journal of Botany, 73, 1168 1177.

Mikesell J (1988) . Comparative development of viable and aborted 
ovules in Phytolacca americana L. (Phytolaccaceae). Botanical Gazette, 149, $196-202$.

Mohana GS, Uma Shaanker R, Ganeshaiah KN, Dayanandan S (2001). Genetic relatedness among developing seeds and intra fruit seed abortion in Dalbergia sissoo (Fabaceae). American Journal of Botany, 88, 1181 - 1188 .

Moody-Weis JM, Heywood JS (2001) . Pollination limitation to reproductive success in the Missouri evening primrose, Oenothera macrocarpa (Onagraceae). American Journal of Botany, 88 , $1615-1622$.

Nicholls MS (1987). Spatial pattern of ovule maturation in the inflorescence of Echium vulgare: demography, resource allocation and the constraints of architecture. Biological Journal of the Linnean Society, 31, $247-256$.

Niesenbaum RA (1996) . Linking herbivory and pollination: defoliation and selective fruit abortion in Lindera benzoin. Ecology, $77,2324-2331$

Niesenbaum RA (1999). The effects of pollen load size and donor diversity on pollen performance, selective abortion, and progeny vigor in Mirabilis jalapa (Nyctaginaceae). American Journal of Botany, 86, $261-268$.

Obeso JR (1993). Selective fruit and seed maturation in Asphodelus albus Miller (Liliaceae). Oecologia, 93, 564-570.

Petersen CE, Lindsey SC, Dudgeon DM, Pertell RA (1998). The effect of seed predation on pod abortion by the prairie legume, Baptisia leucophaea. Transactions of the Illinois State Academy of Science, 91, $47-52$.

Quesada M, Winsor JA, Stephenson AG (1996). Effects of pollen competition on the reproductive performance in cucurbit hybrids (Cucurbitaceae) : $F_{1}$ and backcross generations. Canadian Journal of Botany, 74, $1113-1118$.

Raspé O, Guillaume P, Jacquemart AL (2004) . Inbreeding depression and biased paternity after mixed-pollination in Vaccinium myrtillus L. (Ericaceae). International Journal of Plant Sciences, $165,765-771$.

Rocha OJ, Aguilar G (2001). Reproductive biology of the dry forest tree Enterolobium cyclocarpum (guanacaste) in Costa Rica: a comparison between trees left in pastures and trees in continuous forest. American Journal of Botany, 88, 1607 - 1614.

Rocha OJ, Stephenson AG (1990). Effect of ovule position on seed production, seed weight, and progeny performance in Phaseolus coccineus L. (Leguminosae). American Journal of Botany, 77, $1320-1329$.

Skogsmyr I, Lankinen A (1999) . Selection on pollen competitive ability in relation to stochastic factors influencing pollen deposition. Evolutionary Ecology Research, 1, 971 - 985.

Skogsmyr I, Lankinen $\AA$ (2002). Sexual selection: an evolutionary force in plants? Biological Reviews, 77, 537 - 562 .

Snow AA, Spira TP (1991). Differential pollen-tube growth rates and nonrandom fertilization in Hibiscus moscheutos (Malvaceae). American Journal of Botany, 78, 1419 - 1426.
Stephenson AG (1980). Fruit set, herbivory, fruit production, and the fruiting strategy of Catalpa speciosa (Bignoniaceae). Ecolo$g y, 61,57-64$.

Stephenson AG (1981). Flower and fruit abortion: proximate causes and ultimate functions. Annual Review of Ecology and Systematics, $12,253-279$.

Stephenson AG, Winsor JA (1986). Lotus corniculatus regulates offspring quality through selective fruit abortion. Evolution, 40, $453-458$.

Susko DJ, Lovett-Doust L (1998). Variable patterns of seed maturation and abortion in Alliaria petiolata (Brassicaceae). Canadian Journal of Botany, 76, 1677 - 1686.

Sutherland S, Delph LF (1984). On the importance of male fitness in plants: patterns of fruit-set. Ecology, 65, 1093 - 1104 .

Sutherland S (1986). Patterns of fruit-set: what controls fruit-flower ratio in plants? Evolution, 40, $117-128$.

Thompson JD, Dommée B (1993). Sequential variation in the components of reproductive success in the distylous Jasminum fruticans (Oleaceae). Oecologia, 94, 480-487.

Torres C, Eynard MC, Aizen MA, Galetto L (2002). Selective fruit maturation and seedling performance in Acacia caven (Fabaceae). International Journal of Plant Sciences, 163, $809-813$.

Uma Shaanker R, Ganeshaiah KN, Bawa KS (1988). Parent offspring conflict, sibling rivalry and brood size patterns in plants. Annual Review of Ecology and Systematics, 19, 177 - 205.

Vaughton G (1993). Nonrandom patterns of fruit set in Banksia spinulosa (Proteaceae) : interovary competition within and among inflorescences. International Journal of Plant Sciences, 154, $306-313$.

Vaughton G, Ramsey M (2003). Self-compatibility and floral biology in subdioecious Wurmbea dioica (Colchicaceae). Australian Journal of Botany, 51, $39-45$.

Verdú1 M, García-Fayos P (1998). Ecological causes, function, and evolution of abortion and parthenocarpy in Pistacia lentiscus (Anacardiaceae) . Canadian Journal of Botany, 76, $134-141$.

Wang KS (2003). Relationship between empty seed and genetic factors in European beech (Fagus sylvatica L.). Silva Fennica, $37,419-428$.

Wang YC (王迎春), Hou YW(侯艳伟), Zhang YJ(张颖娟), Yang C (杨持) (2001). Reproductive strategies of Tetraena mongolica Maxim. Acta Phytoecologica Sinica (植物生态学报), 25, 699 - 703. (in Chinese with English abstract)

Webb CJ, Bawa KS (1985). Patterns of fruit and seed production in Bauhinia ungulata (Leguminosae). Plant Systematics and Evolution, 151, 55-65.

Webb CJ, Shand JE (1985). Reproductive biology of tree lucerne (Chamaecytisus palmensis, Leguminosae). New Zealand Journal of Botany, 23, $597-606$.

Wesselingh RA, Arnold ML (2003) . A top-down hierarchy in fruit set on inflorescences in Iris fulva (Iridaceae). Plant Biology, 5, 
$651-660$.

Willson MF, Price PW (1980). Resource limitation of fruit and seed production in some Asclepias species. Canadian Journal of Botany, 58, $2229-2233$.

Winsor JA, Peretz S, Stephenson AG (2000). Pollen competition in a natural population of Cucurbita foetidissima ( Cucurbitaceae) . American Journal of Botany, 87, 527 - 532 .

Wyatt R (1980). The reproductive biology of Asclepias tuberosa. I . Flower number, arrangement, and fruit-set. New Phytologist, $85,119-131$.

Wyatt R (1982). Inflorescence architecture: how flower number, arrangement, and phenology affect pollination and fruit-set. American Journal of Botany, 69, 585 - 594 .
Xiao YA (肖宜安), Zeng JJ (曾建军), Li XH(李晓红), Hu WH (胡文海), He P (何平) (2006). Pollen and resource limitations to lifetime and seed production in a wild population of the endangered plant Disanthus cercidifolius Maxim. var. longipes H. T. Chang (Hamamelidaceae) . Acta Ecologica Sini$c a$ (生态学报 $), 26,496-502$. (in Chinese with English abstract)

Yang CF, Sun SG, Guo YH (2005). Resource limitation and pollen source (self and outcross) affecting seed production in two louseworts, Pedicularis siphonantha and P. longiflora (Orobanchaceae) . Botanical Journal of the Linnean Society, 147, 83 89. 